\title{
The effects of the inclination of the head and focal length on psychological and physiological states
}

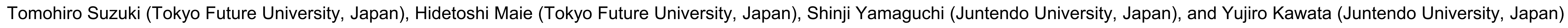

\section{Introduction}

-The body and mind have a reciprocal effect on each other. In fact, the literature has presented evidence that physical posture influences emotion and cognition.

-The various aspects of posture have been investigated in conventional research paradigms, and head inclination is one of these elements. Previous studies have examined and supported the effects of emotion on posture, including the possible effects of head inclination.

-The effects of head inclination on emotion have also been examined directly. Such studies generally suggested that downward head inclination exerted negative effects, while upward head inclination exerted positive effects on psychological and physiological states.

-In previous studies, when head inclination or postures are treated as variables, the focal length from the closest object in the range of view was not considered. Therefore, the effects of both head inclination and focal length may have been confounded.

-The current study examined whether the focal length to an object that blocks one's sight influences one's psychological and physiological states as it interacts with the relevant head inclination simultaneously.

\section{Materials and Methods}

\subsection{Participants}

Twenty college students ( 6 men and 14 women; mean age $=19.85, S D=1.04$

years) participated in the experiment.

\subsection{Procedure}

The study variables were two focal-length conditions (long or short) $\mathrm{X}$ two inclination-of-the head conditions (upward or downward).

\subsection{Apparatus}

A cubic frame, which was $4 \mathrm{~m}$ in height, $1.8 \mathrm{~m}$ in depth, and $1.8 \mathrm{~m}$ in width was constructed, and a white cloth was attached to the eye level of each participant to block his or her view (Appendix 1)

\subsection{Measurements}

\subsubsection{Physiological state}

To measure the participants' physiological states, amylase levels in their saliva were measured.

\subsubsection{Psychological state}

To measure the extent to which they felt the emotions, a pen-and-pencil self-report questionnaire was administered. Responses ranged from 0 (Not at all) to 5 (Very much).

In addition, the two emotional spectrums ("high arousal vs. low arousal" and "pleasure vs. displeasure") were placed over the Visual Analogue Scale (VAS) to assess current mental status (Appendix 2).

\section{Results}

The means and $S D$ s for the amylase levels, questionnaire, and VAS scores measured in each condition, and results of within-subjects two-way ANOVAs are shown in Table 1.

Table 1 The means and SDs of each variable in each condision and results of ANOVAs

\begin{tabular}{|c|c|c|c|c|c|c|c|c|c|c|c|}
\hline & \multicolumn{4}{|c|}{ upward } & \multicolumn{4}{|c|}{ downward } & \multicolumn{3}{|c|}{ ANOVA } \\
\hline & \multicolumn{2}{|c|}{ long } & \multicolumn{2}{|c|}{ short } & \multicolumn{2}{|c|}{ long } & \multicolumn{2}{|c|}{ short } & \multirow{2}{*}{ focal length } & \multirow{2}{*}{ head inclinaation } & \multirow{2}{*}{ interaction effec } \\
\hline & $M$ & $S D$ & $M$ & $S D$ & $M$ & $S D$ & $M$ & $S D$ & & & \\
\hline amylase level & 12.90 & 9.43 & 16.30 & 10.27 & 15.95 & 10.55 & 13.00 & 6.22 & $F(1,19)=0.02$ & $F(1,19)=0.00$ & $F(1,19)=3.44$ \\
\hline stifling & 0.85 & 1.31 & 2.65 & 1.42 & 1.10 & 0.91 & 2.25 & 1.65 & $F(1,19)=26.47 * * *$ & $F(1,19)=0.06$ & $F(1,19)=1.94$ \\
\hline anxiety & 1.95 & 1.54 & 2.35 & 1.27 & 1.65 & 1.27 & 2.20 & 1.40 & $F(1,19)=2.16$ & $F(1,19)=0.53$ & $F(1,19)=0.12$ \\
\hline vgorousness & 1.15 & 0.59 & 1.55 & 0.69 & 2.00 & 1.21 & 1.65 & 0.93 & $F(1,19)=0.03$ & $F(1,19)=4.97 *$ & $F(1,19)=3.83$ \\
\hline nervousness & 2.05 & 1.50 & 2.05 & 1.43 & 1.40 & 1.05 & 2.30 & 1.34 & $F(1,19)=2.30$ & $F(1,19)=0.51$ & $F(1,19)=4.98 *$ \\
\hline unquietness & 2.10 & 1.33 & 2.45 & 1.64 & 1.50 & 1.05 & 2.20 & 1.64 & $F(1,19)=2.34$ & $F(1,19)=1.73$ & $F(1,19)=0.49$ \\
\hline concentration & 2.60 & 1.19 & 2.45 & 0.94 & 2.55 & 1.15 & 2.70 & 0.80 & $F(1,19)=0.00$ & $F(1,19)=0.23$ & $F(1,19)=0.90$ \\
\hline stress & 1.50 & 1.28 & 2.65 & 1.42 & 1.10 & 0.85 & 2.10 & 1.25 & $F(1,19)=19.84^{* * *}$ & $F(1,19)=4.34$ & $F(1,19)=0.19$ \\
\hline relaxation & 2.50 & 1.36 & 2.05 & 1.15 & 3.45 & 0.83 & 2.20 & 1.11 & $F(1,19)=17 \cdot 11^{* * *}$ & $F(1,19)=3.54$ & $F(1,19)=2.85$ \\
\hline high arousal & 1.25 & 6.08 & -0.15 & 4.86 & 1.70 & 5.55 & -1.60 & 4.89 & $F(1,19)=4.59 *$ & $F(1,19)=0.56$ & $F(1,19)=0.22$ \\
\hline pleasure & 0.40 & 4.12 & -0.10 & 4.17 & 2.50 & 2.80 & 0.55 & 3.25 & $F(1,19)=3.34$ & $F(1,19)=2.49$ & $F(1,19)=1.32$ \\
\hline
\end{tabular}

note ${ }^{* * *} p<.001,{ }^{*} p<.05$

\section{Discussion}

The findings indicated that head inclination exerted a partial effect on these states, while focal length to an object that blocked participants' view exerted a significant effect on psychological states.

-Relative to short focal length, long focal length to an object induced positive emotions more readily. If focal length to an object was controlled in both the upward and downward head inclination conditions, the effect of head inclination would not have been detected. Moreover, the current study's findings implied that the effect of head inclination described in the literature could have been confounded with the effect of focal length.

-The current study showed that the effect of head inclination was significant and nonnegligible. Therefore, we could not conclude that all effects of head inclination were confounded with focal length

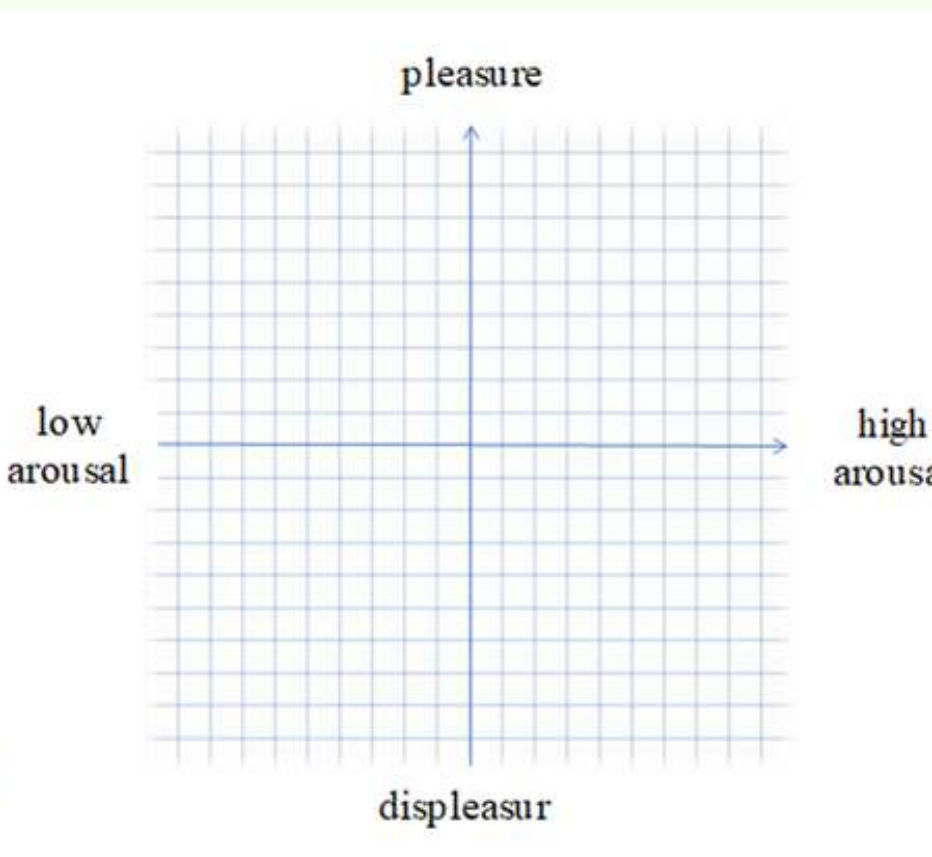

Appendix 2 Tow dimensional VAS
Appendix 2 Tow dimensional VAS

-The current study did not show any effect of head inclination or focal length on physiological states through the analysis of salivary amylase.

-If the current study had also utilized these physiological measures, the effect of head inclination or focal length on

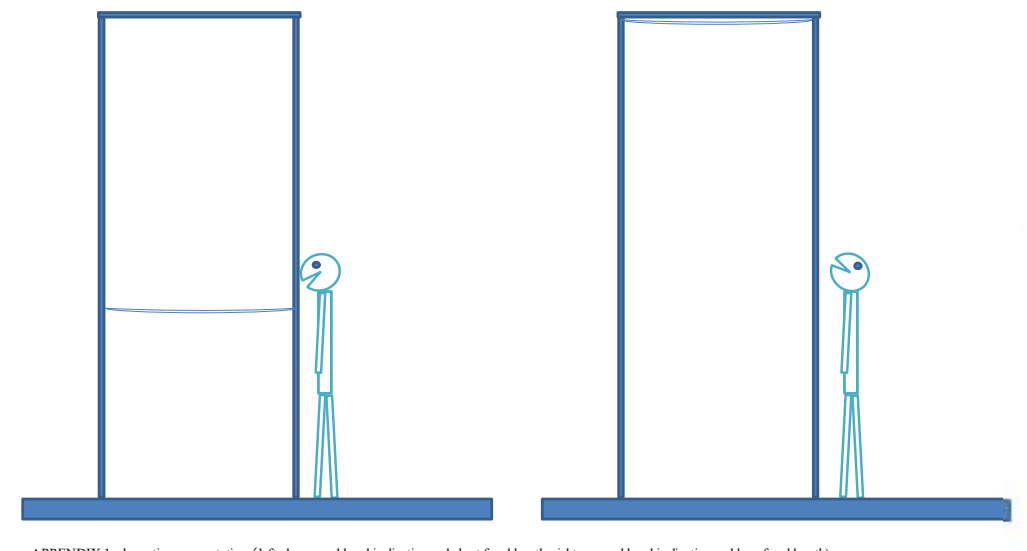

Appendix 1 schematic representations physiological states could have been identified. Therefore, future research should consider various physiological measures.

This study should contribute to the psychology of the mind and body connection by providing new knowledge. 